\title{
SÍNTESE DE SULFADIAZINA E SULFADIAZINA DE PRATA EM ESCALA SEMI-MICRO: PRÁTICA EXPERIMENTAL EM SÍNTESE DE FÁRMACOS
}

Áurea Donizete Lanchote Borges, Gino Del Ponte, Alberto Federman Neto e Ivone Carvalho*

Departamento de Ciências Farmacêuticas, Faculdade de Ciências Farmacêuticas de Ribeirão Preto, Universidade de São Paulo, Av. Zeferino Vaz, s/n, 14040-903 Ribeirão Preto - SP

Recebido em 28/7/04; aceito em 29/10/04; publicado na web em 28/2/05

\begin{abstract}
SYNTHESIS OF SULFADIAZINE AND SILVER SULFADIAZINE IN SEMI-MICRO SCALE, AS AN EXPERIMENTAL PRACTICE IN DRUG SYNTHESIS. The total synthesis of sulfadiazine and silver sulfadiazine from readily available starting materials was adapted to semi-micro laboratory scale and is proposed as an experiment in drug synthesis for undergraduate courses.
\end{abstract}

Keywords: drug synthesis; sulfonamide drugs; sulfadiazine.

\section{INTRODUÇÃO}

A descoberta da atividade antibacteriana das sulfas foi oficializada em 1935 com a publicação do trabalho "Uma Contribuição à Quimioterapia das Infecções Bacterianas", atividade biológica da $p$-sulfamidocrisoidina (Prontosil Rubrum ${ }^{\circledR}$, 1), pelo patologista e bacteriologista alemão Gerhardt Domagk. Esta substância havia sido sintetizada em 1932 por Mietsch e Klarer (Bayer), com base na química clássica de corantes têxteis, especificamente para ser testada como antibacteriano ${ }^{1}$. O crédito por essa descoberta deu a Domagk o prêmio Nobel de Medicina em $1939^{3}$.

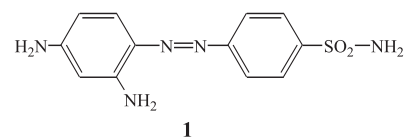

A partir desta época foram surgindo outras substâncias antimicrobianas, algumas obtidas sinteticamente e outras isoladas de microorganismos como a penicilina, descoberta por Fleming em 1928 , e que demonstraram ter acentuada ação antibacteriana ${ }^{4}$.

Forneau e colaboradores ${ }^{5}$, analisando metabólitos no sangue e urina de pacientes em tratamento com Prontosil ${ }^{\circledR}$, detectaram a presença de sulfanilamida (substância conhecida desde 1908) . $^{6}$ Concluiu-se através deste estudo que a parte ativa da molécula era a sulfanilamida e que os vários quimioterápicos antibacterianos até então bem conhecidos, somente agiam devido à presença do grupo farmacofórico sulfonamídico, cujo mecanismo de ação foi posteriormente elucidado e relacionado à inibição da enzima diidropteroato sintase bacteriana.

Em 1938 surgiram as primeiras sulfas heterocíclicas, como sulfapiridina (2) e sulfatiazol (3), apresentando atividade antibacteriana de amplo espectro e superioridade terapêutica ${ }^{1}$. Por serem relativamente tóxicas, alguns pesquisadores direcionaram suas pesquisas para a busca de outros antibacterianos não sulfonamídicos, conduzindo à obtenção de nitrofurantoína (4) e nitrofurazona $(\mathbf{5})^{1,7}$, derivados do furfural nitro-substituído, depois abandonados para uso sistêmico, por sua menor eficácia aliada à uma toxidez que se revelou muito maior que a encontrada nas sulfas e nos antibióticos ${ }^{8}$.

*e-mail: carronal@usp.br<smiles>Nc1ccc(ONc2ccccn2)cc1</smiles>

2<smiles>O=C1CN(/N=C/c2ccc([N+](=O)[O-])o2)C(=O)N1</smiles>

Em 1940 surgiram as sulfas com núcleos pirimidínicos, dentre elas a sulfadiazina $(\mathbf{6})^{1,9}$. As sulfas pirimidínicas foram utilizadas no combate contra infecções causadas por cocos e colibacilos. O sal de prata da sulfadiazina (7), introduzido na prática médica corrente por Fox e colaboradores ${ }^{10}$ em 1968, é hoje um medicamento tópico útil para o tratamento de queimaduras e úlceras varicosas, por proporcionar cicatrização rápida.

A partir do final da década de 40 os antibióticos tenderam a substituir as sulfas na quimioterapia devido à sua menor toxidez $\mathrm{e}$ maior espectro de ação ${ }^{1}$.<smiles>Cc1cc(NOc2ccc(N)cc2)no1</smiles><smiles>COc1cc(Cc2cnc(N)nc2N)cc(OC)c1OC</smiles>

Após a observação de que certas bactérias adquiriam resistência causada pelos antibióticos, renasceu o interesse pelas sulfas e a pesquisa de novos derivados sulfonamídicos, estimulados pela facilidade de obtenção e baixo custo.

Atualmente, algumas sulfas são utilizadas na forma associada, 
como por ex. sulfametoxazol (8) e trimetroprim (9) $\left(\right.$ Bactrim $\left.^{\circledR}\right)$ para potencializar seus efeitos. Além disso, novos fármacos derivados de sulfa têm sido bastante promissores como agentes antitumorais ${ }^{11}$.

Um dos principais objetivos do curso experimental de Síntese de Fármacos é ensinar a obtenção de substâncias de uso terapêutico corrente em poucas etapas, com possibilidade de interrupções temporárias sem perdas qualitativas ou quantitativas por degradação dos intermediários e do produto final, com disponibilidade de reagentes e adequação de carga horária média semanal de $10(5+5)$ h.

\section{RESULTADOS E DISCUSSÃO}

As aulas práticas foram planejadas com o objetivo de proporcionar aos alunos a (i) sedimentação de técnicas e conceitos básicos oriundos das disciplinas (pré-requisitos) de Química Orgânica Experimental, Química Analítica Qualitativa e Quantitativa e Química Farmacêutica; (ii) manipulação laboratorial em escala semimicro de materiais e reagentes de uso clássico e de maior disponibilidade e (iii) acompanhamento de toda trajetória do desenvolvimento de fármaco envolvendo síntese, isolamento, purificação, caracterização dos intermediários-chave e do produto final, análise farmacêutica e padronização por métodos oficiais de farmacopéias. Nessa disciplina, todos os resultados obtidos foram interpretados e discutidos durante as aulas.

Pelas razões delineadas acima, foram elaborados experimentos em escala semi-micro para preparações de sulfadiazina e sulfadiazina de prata ${ }^{12-16}$, uma vez que sua síntese envolve vários tipos de reações orgânicas, como condensação, acetilação, proteção e desproteção de grupos funcionais e clorossulfonaçãa ${ }^{17-21}$. Deste modo, considerou-se que o aluno teve um treinamento adequado e que foi dada a oportunidade para adquirir uma visão geral da metodologia sintética em múltiplas etapas.

Como parte do aprendizado, os alunos foram avaliados no final do curso pela apresentação de seminários com temas relacionados a metodologias sintéticas, técnicas de purificação, análises espectrofotométricas e cromatográficas, bem como por relatórios.

O principal processo para a obtenção de sulfadiazina envolveu o método geral (Figura 1) de síntese de sulfas, ou seja, de condensação entre cloreto de 4-(acetilamino)benzenossulfonila (10) e 2aminopirimidina (11). Existem algumas modificações desta metodologia descritas na literatura que estão relacionadas ao solvente, reagentes, temperatura e tempo de reação para processos industriais e laboratoriais ${ }^{9,12-21}$.

Com base nesses dados, foi desenvolvida uma metodologia adequada e relativamente simples para a preparação de 6 e 7 no curso de Síntese de Fármacos. A rota sintética está ilustrada na Figura 1.

Anilina (12) comercial foi empregada como material de partida e convertida, em uma primeira etapa, ao correspondente derivado acetanilida (13), para proteção do grupamento amino. Vários tipos de reação de acilação de aminas aromáticas descritas em livros de Química Orgânica Experimental ${ }^{17-20}$ foram testados e comparados, como em fase aquosa não catalisada ou catalisada por bases inorgânicas "Schotten-Baumann"; acetilação com ácido acético a refluxo (o primeiro método de acilação conhecido, ainda hoje importante do ponto de vista industrial); acetilação clássica com anidrido acético em excesso ou catalisada por ácido sulfúrico concentrado ou ácido ortofosfórico, etc ${ }^{17,22}$.

A acetilação a frio em meio aquoso com anidrido acético e acetato de sódio como base foi o procedimento de escolha devido às vantagens de rendimento $(70-90 \%)$ e pureza de $\mathbf{1 3}$ obtida $^{17,19}$. As impurezas escuras do reagente $\mathbf{1 2}$ foram removidas pela sua disso-

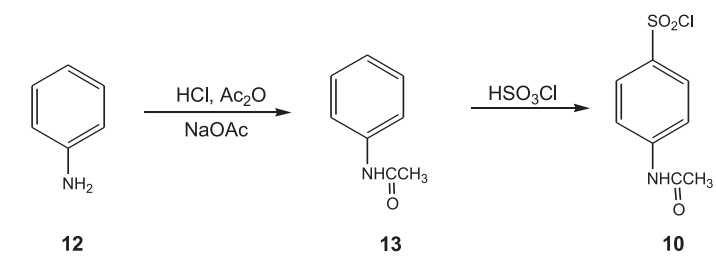

$2(\mathrm{MeO})_{2} \mathrm{CHCH}_{2} \mathrm{CH}(\mathrm{OMe})_{2}+1\left[\mathrm{H}_{2} \mathrm{NC}(=\mathrm{NH}) \mathrm{NH}_{2}\right]_{2} \cdot \mathrm{H}_{2} \mathrm{CO}_{3} \longrightarrow 15$
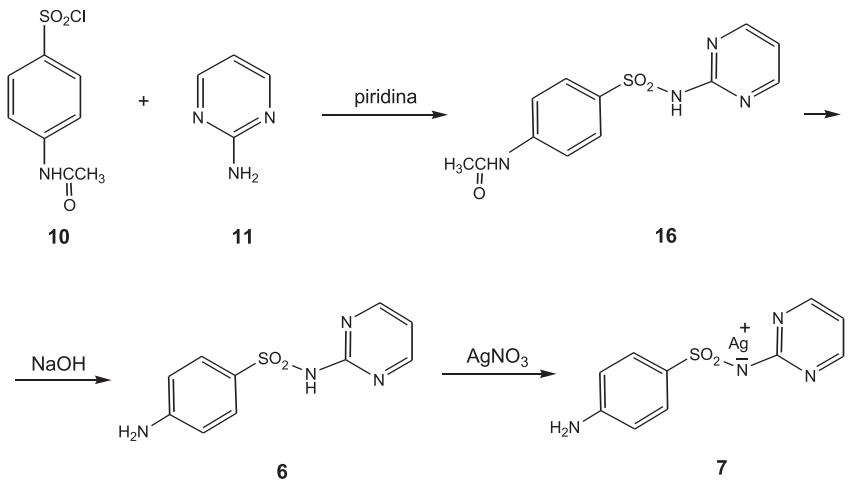

Figura 1. Síntese de Sulfadiazina (6) e Sulfadiazina de Prata (7)

lução em forma de cloridrato e tratamento com carvão ativo (ver parte experimental).

O composto $\mathbf{1 3}$ foi tratado com ácido clorossulfônico originando o intermediário-chave comum da síntese de sulfas, composto 10. Pela importância industrial das sulfas, a reação de clorossulfonação de $\mathbf{1 3}$ foi muito bem investigada e descrita na literatura $^{14-21}$. O melhor resultado foi obtido a partir do espalhamento de 13 seco e fundido no frasco reacional, seguido de solidificação e introdução de ácido clorossulfônico. A liberação de vapores tóxicos de $\mathrm{HCl}$ foi evitada pelo seu borbulhamento em solução aquosa de $\mathrm{NaOH}$.

Apesar de $\mathbf{1 0}$ ser descrito como um sal pouco estável, nos experimentos realizados observadou-se que este pode ser seco em dessecador e armazenado em freezer por período prolongado, sem decomposição apreciável. Sua estocagem é interessante, uma vez que se trata de intermediário-chave para a síntese de várias outras sulfas, como sulfanilamida, sulfaguanidina, sulfapiridina, sulfatiazol, sulfametoxazol etc ${ }^{12-21}$.

O procedimento clássico para síntese de diferentes sulfas envolve a condensação de $\mathbf{1 0}$ com diversas aminas funcionalizadas. No caso da sulfadiazina, o correspondente derivado amino é a 2aminopirimidina (11). Esta amina pode ser preparada por diversos processos, por ex., reação de álcool propargílico e cloridrato de guanidina $^{23}$ ou dicianoamida ${ }^{24}$ em solução aquosa ácida; hidrólise de tetraalcoxipropano para formar malonaldeído, seguida de condensação com cloridrato de guanidina ${ }^{25}$, redução de cloropirimidinas $^{26}$ e vários outros métodos ${ }^{9}$.

Entre os diversos métodos testados, o mais conveniente foi a condensação de Träube ${ }^{27,28}$, entre tetrametoxipropano (14) e sal de guanidina (15) em solução ácida, baseada no protocolo descrito por Kobayashi ${ }^{29}$, devido à sua facilidade de manipulação. O sal nitrato de guanidina está descrito como o reagente que fornece alto rendimento ${ }^{15}$, contudo, o sal carbonato foi utilizado por sua disponibilidade comercial e segurança no laboratório ${ }^{30}$ (Figura 1). 
A reação de condensação de $\mathbf{1 0}$ e $\mathbf{1 1}$ foi realizada inicialmente na presença de $\mathrm{NaOH}$ aquoso, no entanto, o baixo rendimento do produto levou a uma adaptação das técnicas desenvolvidas por Roblin Jr. e colaboradores ${ }^{9}$ e Sittig ${ }^{31}$. A reação efetuada na presença de piridina anidra forneceu acetilsulfadiazina bruta (16). Devido às dificuldades envolvidas na sua purificação, esta foi usada diretamente na etapa posterior de hidrólise alcalina para remoção do grupo protetor $N$-acetila e obtenção de 6 (20-40\%, 2 etapas) ${ }^{9}$. Visando obter sulfadiazina de pureza grau farmacêutico, esta foi recristalizada diversas vezes até que o grau de pureza estivesse de acordo com os testes oficiais de monografias de farmacopéias, usando sulfadiazina Aldrich como referência ${ }^{32,33}$.

Sulfadiazina de prata (7) foi obtida pela simples reação entre 6 e nitrato de prata ${ }^{34}$. Pela sua quase total insolubilidade, deve ser preparada com sulfadiazina previamente purificada, pelo menos recristalizada uma vez em acetonitrila/água.

\section{PARTE EXPERIMENTAL}

Tetrametoxipropano (Aldrich), carbonato de guanidina (Sardi), anilina (Vetec), ácido clorossulfônico (Aldrich), etc. foram obtidos comercialmente e utilizados sem purificação prévia. Piridina (Merck) foi seca sobre $\mathrm{KOH}$ sólido e destilada sob pressão atmosférica. O sal de guanidina pode também ser preparado, embora com rendimento moderado, por pirólise seca de tiocianato de amônio $^{30,35}$.

Os espectros vibracionais na região do infra-vermelho foram registrados em espectrofotômetro Perkin-Elmer 1420, em pastilhas de $\mathrm{KBr}$. O aparelho foi calibrado usando a banda padrão de transmitância de filme de poliestireno a $1601 \mathrm{~cm}^{-1}$. Os espectros de RMN de hidrogênio foram feitos em espectrômetro Bruker AC-80, usando tetrametilsilano (TMS) como padrão interno.

A análise por cromatografia gasosa foi realizada em cromatógrafo Hewlett-Packard 5890-II, coluna HP-5 (5\% de polímero cruzado de siloxano), $30 \mathrm{~m}$ x $0,25 \mu_{\mathrm{n}}$. Condições de análise: solvente acetato de etila, temperatura inicial da coluna $80{ }^{\circ} \mathrm{C}$ e final $210{ }^{\circ} \mathrm{C}$, rampa de aquecimento $6 \mathrm{~min}$. Temperatura: injetor $220^{\circ} \mathrm{C}$ e detector $305^{\circ} \mathrm{C}$.

Os pontos de fusão não corrigidos foram medidos em aparelho tipo Kofler, Thermolyne $703 \mathrm{~A}$, termômetro -10 a $300{ }^{\circ} \mathrm{C}$, aferido com ácido benzóico P.A. Merck (Pf: 122,6 a $\left.123,1{ }^{\circ} \mathrm{C}\right)^{36}$.

\section{Síntese}

Preparação de 2-aminopirimidina (11), via condensação de Träube

Em balão de $100 \mathrm{~mL}$, mergulhado em banho de gelo, foram adicionados solução de $\mathrm{H}_{2} \mathrm{SO}_{4} 50 \%$ v/v $(43 \mathrm{~mL})$, carbonato de guanidina (15) $(2,7 \mathrm{~g}, 15 \mathrm{mmol})$, tetrametoxipropano $(\mathbf{1 4})(6,6 \mathrm{~mL}$, $40 \mathrm{mmol})$. A mistura reacional foi agitada a temperatura ambiente por $10 \mathrm{~h}$ e alcalinizada lentamente, inicialmente com $\mathrm{NaOH}$ sólido e, no final da neutralização, com solução aquosa de $\mathrm{NaOH} 30$ $40 \%$, até obtenção de $\mathrm{pH} 9,0$, com resfriamento em gelo para evitar aquecimento. Esta etapa é bastante crítica, o aquecimento da mistura durante a neutralização pode causar o seu escurecimento (marrom) e diminuir o rendimento, com formação de polímeros. O produto foi extraído com acetato de etila ( 3 x $20 \mathrm{~mL}$ ), e a fase orgânica foi seca com $\mathrm{Na}_{2} \mathrm{SO}_{4}$ anidro, filtrada e o solvente evaporado. Após secagem em dessecador o composto $\mathbf{1 1}$ foi obtido com $70-88 \%$, como cristais amarelos ou alaranjados. Pf.: $123-126{ }^{\circ} \mathrm{C}$ (lit. ${ }^{9} 125-127^{\circ} \mathrm{C}$ ); CG: tempo de retenção de 4,75 min com $90 \%$ de pureza; $\mathrm{CCD}: \mathrm{Rf}=0,29$, com eluente $\mathrm{n}$-hexano/acetato de etila 7:3; IV (KBr, cm $\left.{ }^{-1}\right): v_{\max } 3360$ e $3180(\mathrm{~N}-\mathrm{H}), 1650(\mathrm{~N}-\mathrm{H}), 1570$ e $1550(\mathrm{C}=\mathrm{C}$, anel heteroaromático), $1475(\mathrm{C}=\mathrm{N}), 805(\mathrm{C}-\mathrm{H}$ hetero- aromático), $\mathrm{RMN}\left(80 \mathrm{MHz}, \mathrm{CDCl}_{3}\right): \delta_{\mathrm{H}} 5,5-6,0\left(\mathrm{~s}, \operatorname{largo}, 2 \mathrm{H}, \mathrm{NH}_{2}\right)$, 6,6 (t, 1H, H-5), 8,3 (d, 2 H, H-4 e H-6). Os espectros obtidos correspondem aos do catálogo de padrões internacionais, (medidos com amostras autênticas), SDBS nº $3558^{37}$.

\section{Preparação de acetanilida (13), reação de acetilação}

Em erlenmeyer de $125 \mathrm{~mL}$, contendo $\mathrm{H}_{2} \mathrm{O}(50 \mathrm{~mL})$, foram adicionados $\mathrm{HCl}$ concentrado $(2,17 \mathrm{~mL}, 22 \mathrm{mmol})$, anilina (12) $(2,37 \mathrm{~mL}, 22 \mathrm{mmol})$ e a mistura foi agitada até completa dissolução da anilina. Carvão ativo $(0,1 \mathrm{~g})$ foi introduzido e a mistura aquecida por $10 \mathrm{~min}$ a $50-60{ }^{\circ} \mathrm{C}$ e filtrada à quente. $\mathrm{O}$ filtrado foi resfriado em banho de gelo, e à solução gelada, foi adicionado anidrido acético $(3,03 \mathrm{~mL}, 32 \mathrm{mmol})$ em uma única porção (adição gradual pode diminuir o rendimento). A mistura foi agitada e, em seguida, foi introduzido solução de acetato de sódio tri-hidratado (3,9 g, 29 mmol, em $12 \mathrm{~mL}$ de $\left.\mathrm{H}_{2} \mathrm{O}\right)$, em uma única porção. Após agitação vigorosa para formação de sólido (agitação manual), a mistura foi mantida por 30-45 min em banho de gelo, filtrada a vácuo, lavada com pequena quantidade de $\mathrm{H}_{2} \mathrm{O}$ gelada e seca ao ar. O composto 13 foi obtido com rendimento de 70-90\%, como cristais incolores e inodoros. Pf. $113-116{ }^{\circ} \mathrm{C}$ (lit. ${ }^{16-18,38} 113-115^{\circ} \mathrm{C}$ ); CG: tempo de retenção de 15,7 min com $86 \%$ de pureza, CCD: Rf= 0,29 com eluente: n-hexano/acetato de etila 7:3; IV $\left(\mathrm{KBr}, \mathrm{cm}^{-1}\right)$ : $v_{\max } 3300$ e $3180(\mathrm{~N}-\mathrm{H}) ; 1660(\mathrm{C}=\mathrm{O}) ; 1600(\mathrm{C}=\mathrm{C}$, anel aromático); $1320(\mathrm{C}-\mathrm{H}) ; 1260(\mathrm{C}-\mathrm{H}$ e C-N); RMN (80 MHz, CDCl $)$ ): $\delta_{\mathrm{H}} 2,1(\mathrm{~s}$, $3 \mathrm{H}, \mathrm{CH}_{3}$ ); 7,0-7,6 (m, $\left.5 \mathrm{H}, \mathrm{Ar}-\mathrm{H}\right) ; 8,3$ (sl, $\left.1 \mathrm{H}, \mathrm{N}-\mathrm{H}\right)$. Os espectros estão em concordância com o catálogo de padrões internacionais ${ }^{37}$, SDBS $n^{0} 729$. Os cristais obtidos foram secos ao ar ou em estufa a $60{ }^{\circ} \mathrm{C}$. A recristalização foi necessária apenas quando anilina impura foi empregada e o tratamento com carvão ativo foi omitido. A recristalização de acetanilida foi realizada em um volume mínimo de metanol/água 1:10 fervente, filtrada, resfriada em banho de gelo e filtrada a vácuo.

Preparação de cloreto de 4-(acetilamino)benzenossulfonila (10), reação de clorossulfonação

Em capela de exaustão, foram aquecidos acetanilida (13) (2,0 g, $15 \mathrm{mmol}$ ) em balão de $50 \mathrm{~mL}$ (banho de silicone), até fundir totalmente o sólido (temperatura aproximada de $130{ }^{\circ} \mathrm{C}$ ). $\mathrm{O}$ balão foi lentamente girado para formação de uma película fina até, no máximo, metade da altura da parede do balão, resfriado em banho de gelo. Ácido clorossulfônico $(5,2 \mathrm{~mL}, 78 \mathrm{mmol}$ ) (composto muito corrosivo e fumegante) foi adicionado em uma única porção, e o sistema foi imediatamente conectado a um condensador de refluxo, ligado previamente a um sistema de saída de $\mathrm{HCl}$ gasoso (Figura 2). Os vapores ácidos foram absorvidos em solução de hidróxido de sódio $2 \mathrm{M}$ e a solução alcalina neutralizada antes do descarte. A mistura foi agitada magneticamente por 15 min à temperatura ambiente, aquecida a $60{ }^{\circ} \mathrm{C}$ por mais 15 min e vertida cuidadosamente (hidrólise exotérmica!) sobre gelo (36 g), contido num béquer de $250 \mathrm{~mL}$, ainda em capela de exaustão, lavando o balão com água gelada. Após filtração a vácuo do sólido formado e secagem ao ar, o cloreto $\mathbf{1 0}$ foi isolado como um cristal incolor pouco estável, mas adequadamente estocado em freezer, com rendimento de $70-80 \%$ e de odor característico desagradável. Pf.: 139$148{ }^{\circ} \mathrm{C}$ (lit.161-163 ${ }^{\circ} \mathrm{C}^{17}, 143-148{ }^{\circ} \mathrm{C}{ }^{16,18}$ ). O ponto de fusão pode sofrer alteração com a variação da velocidade de aquecimento. IV $\left(\mathrm{KBr}, \mathrm{cm}^{-1}\right): v_{\max } 3300$ e $3260(\mathrm{~N}-\mathrm{H}) ; 3180$ e $3100(\mathrm{C}-\mathrm{H}$ aromático); 2700 (C-H alifático); $1680(\mathrm{C}=\mathrm{O})$; 1580 (C=C acromático),1530 e 1560 (C-N); 1370 e 1170 ( $\left.\mathrm{SO}_{2} \mathrm{Cl}\right) ; 1000,1030,860$ (def., aromáticos); Espectro em acordo com o padrão internacional, SDBS n ${ }^{0} 6119^{37}$. RMN (80 MHz, DMSO-d $): \delta_{\mathrm{H}} 2,2$ (s, $3 \mathrm{H}$, $\mathrm{CH}_{3}$ ); 7,7 (s, $\left.4 \mathrm{H}, \mathrm{Ar}-\mathrm{H}\right) ; 10,0$ (s largo, $1 \mathrm{H}$ ). 


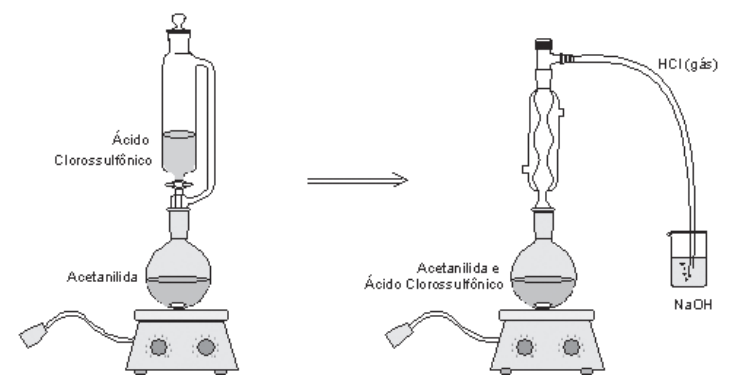

Figura 2. Aparelho utilizado para reação de clorossulfonação

\section{Preparação de acetilsulfadiazina (16)}

Em balão de $50 \mathrm{~mL}$, foram adicionados 2-aminopirimidina (11) $(0,94 \mathrm{~g}, 10 \mathrm{mmol})$, piridina anidra $(3,0 \mathrm{~mL})$ e cloreto de $p$ acetamidobenzenossulfonila (10) $(2,34 \mathrm{~g}, 10 \mathrm{mmol})$. A este sistema foi conectado condensador de refluxo, com tubo dessecante e, após agitação magnética por $4 \mathrm{~h}$ a $55^{\circ} \mathrm{C}$, a mistura foi concentrada a vácuo para remoção de piridina (banho a aproximadamente 40$50{ }^{\circ} \mathrm{C}$ ). Este produto bruto (marrom, viscoso) foi diretamente usado na reação de hidrólise e remoção do grupo protetor, devido às dificuldades de purificação? .

\section{Preparação de sulfadiazina (6), reação de desproteção}

$\mathrm{O}$ produto bruto 16, obtido na reação anterior, foi tratado com solução de $\mathrm{NaOH}$ ( $2 \mathrm{M}, 25 \mathrm{~mL})$. O balão foi acoplado em condensador de refluxo e aquecido por $2 \mathrm{~h}$ a $100{ }^{\circ} \mathrm{C}$. Após este período a mistura foi resfriada até a temperatura ambiente e neutralizada com $\mathrm{HCl}$ concentrado até $\mathrm{pH}$ aproximadamente 6,0 (crítico: sulfadiazina é anfóterica e pode ser solúvel tanto em excesso de bases como de ácidos). A mistura foi resfriada em banho de gelo até a total precipitação do produto, filtrada a vácuo, lavada com pequeno volume de água gelada e seca em estufa a $50{ }^{\circ} \mathrm{C}$. O produto foi recristalizado em mistura acetonitrila/água (2:1) fervente (para cada g de sulfadiazina usou-se aproximadamente $80 \mathrm{~mL}$ de solvente) e o sólido foi filtrado e seco em estufa a $50{ }^{\circ} \mathrm{C}$. Sulfadiazina (6) foi obtida com rendimento em torno de $20-40 \%$, a partir da condensação de $\mathbf{1 0}$ e $\mathbf{1 1}$ (2 etapas), na forma de cristais incolores;. Pf.: $253-265^{\circ} \mathrm{C}$ (lit. $\left.253-257^{\circ} \mathrm{C}^{32,33,38}\right)$. IV $\left(\mathrm{KBr}, \mathrm{cm}^{-1}\right)$ : $v_{\max } 3400$ e $3350(\mathrm{~N}-\mathrm{H}) ; 1650(\mathrm{C}-\mathrm{N}-\mathrm{H}$ angular); $1580(\mathrm{C}=\mathrm{C}$, anel pirimidina); 1320 ( $\left.\mathrm{SO}_{2}\right) ; 1260$ ( $\mathrm{CH}$ pirimidínico e $\mathrm{C}-\mathrm{N}$ anilina). $\mathrm{O}$ espectro obtido corresponde ao descrito na literatura por Sinisterra e colaboredores $^{39}$; RMN (80 MHz, DMSO-d $): \delta_{\mathrm{H}} 5,9$ (s largo, $2 \mathrm{H}$, $\left.\mathrm{NH}_{2}\right) ; 6,6$ (d, 2H, H orto ao grupo amino livre); 7,0 (t, 1H, H-5 do anel pirimidínico), 7,6 (d, 2H, H orto ao grupo sulfonamido), 8,5 (d, 2H, H-4 e H-6 do anel pirimidínico), 11,2 (s largo, 1H, NH).

\section{Preparação de sulfadiazina de prata (7)}

Sulfadiazina (6) (200 mg, 0,80 mmol) foi suspensa em água $(10 \mathrm{~mL})$, aquecida a $60{ }^{\circ} \mathrm{C}$, tratada com $\mathrm{AgNO}_{3}(1 \mathrm{M}, 0,84 \mathrm{~mL}$, corresponde a $0,84 \mathrm{mmol}$ ) e agitada magneticamente por $10 \mathrm{~min}$ a $60{ }^{\circ} \mathrm{C}$. A mistura foi resfriada em gelo e o sal de prata formado foi filtrado, lavado com água gelada e seco por $1 \mathrm{~h}$ a $100{ }^{\circ} \mathrm{C}$ na estufa. O produto 7 foi obtido com rendimento de $70-90 \%$, na forma de cristais amarelo pálido, que escureciam rapidamente quando expostos à luz. Pf $>300{ }^{\circ} \mathrm{C}$ (lit. $.^{32,38}>300{ }^{\circ} \mathrm{C}$ ); IV $\left(\mathrm{KBr}, \mathrm{cm}^{-1}\right): v_{\max }$ 3390 e 3348 (N-H); 1628 (N-H angular); 1580 (C=C, pirimidina); 1418 (vib. característica dos sais de sulfadiazina) ${ }^{39} 1324$ e 1233 (C-N de amina aromática e $\left.\mathrm{SO}_{2}\right) ; 1130$ e $1080(\mathrm{~S}=\mathrm{O})$. A presença das bandas a 1418, 1130 e $1080 \mathrm{~cm}^{-1}$ caracteriza o composto como um sal de sulfadiazina ${ }^{39,40}$. A presença de prata na molécula foi evidenciada pela mineralização de pequena amostra do composto com ácido nítrico concentrado. Após adição de gotas de ácido clorídrico ou de cloreto de sódio diluído, houve formação de precipitado branco de cloreto de prata ${ }^{41}$.

\section{CONCLUSÕES}

Os experimentos seqüenciais realizados pelos alunos foram satisfatórios e forneceram o fármaco sulfadiazina de prata (7) com grau de pureza farmacêutico. As atividades interdisciplinares, envolvendo síntese orgânica, análise espectroscópica, cromatografia e controle de qualidade, contribuíram no processo ensino-aprendizagem desenvolvido no curso de Síntese de Fármacos. O trabalho realizado em escala semi-micro, com emprego de metodologia clássica, reagentes comercialmente disponíveis e possibilidade de interrupções semanais foi conduzido com entusiasmo pelos alunos. No final do curso, no momento de apresentação dos seminários, eles foram capazes de relacionar adequadamente os conceitos teóricos e práticos e demonstraram grande interesse em discutir os métodos empregados, comparando criticamente seus resultados com os demais grupos.

\section{REFERÊNCIAS}

1. Hardman J. G.; Limbird L. E.; Goodman and Gilman's: The Pharmacological Basis of Therapeutics, versão em CD-ROM, 9a ed., McGraw-Hill: New York, 1996; Mitscher, L. A. Em Foye's Principles of Medicinal Chemistry; Williams, D. A.; Lemke, T. L., eds.; $5^{\text {a }}$ ed., Lippincott Williams and Wilkins: Baltimore, 2002, cap. 34.

2. Domagk, G.; Dtsch. Med. Wochenschr. 1935, 61, 250.

3. www.nobel.se/medicine/laureates/1939, acessada em Dezembro 2003.

4. Fleming, A.; Brit. J. Exper. Path. 1929, 10, 22; Wainwright, M.; Perspec. Biol. Med. 2002, 45, 529.

5. Forneau, E.; Tréfouël, J.; Nitti, F.; Bovet, D.; Compt. Rend. Seanc. Soc. Biol. 1936, 122, 652; Buttle, G. A. H.; Gray, W. H.; Stephenson, D.; Lancet 1936, $1,1286$.

6. Gelmo, P.; J. Prakt. Chem. 1908, 77, 369.

7. Julian-Ortiz, J. V. D.; Galvez, J.; Munoz-Collado, C.; Garcia-Domenech, R.; Gimeno-Cardona, C.; J. Med. Chem. 1999, 42, 3308.

8. Black, M.; Rabin, L.; Schatz, N.; Ann. Intern. Med. 1980, 92, 62.

9. Roblin Jr., R. O.; Winnek, P. S.; J. Am. Chem. Soc. 1940, 62, 1999; Roblin Jr., R. O.; Williams, J. H.; Winnek, P. S.; English, J. P.; J. Am. Chem. Soc. 1940, 62, 2002.

10. Fox, C. L.; Arch. Surg. 1968, 96, 184; Fox, C. L.; Rappole, B. W.; Stanford, W.; Surg. Gynecol. Obst. Int. Abstr. Surg. 1969, 128, 1021.

11. Owa, T.; Nagasu, T.; Expert. Opin. Ther. Patol. 2000, 10, 1725.

12. Kleamann, V. A.; Engel, J.; Pharmazeutische Wirkstoffe, Synthese, Patente, Arwendungen, G. Thieme Verlag: Sttutgart, 1978.

13. Lednicer, D., Mitscher, L. A.; The Organic Chemistry of Drug Synthesis., Wiley-Interscience/John Wiley: New York, 1990, vols.1-4.

14. Stewart, J.; J. Chem. Soc. 1922, 121, 2558.

15. Crespo, C. M.; Sintesis Industrial de Medicamentos. Obtencion Industrial, Analisis e Farmacologia de los Medicamentos, Editorial Dossat: Madrid, 1957, p. 288-298.

16. Giral, F.; Productos Quimicos e Farmaceuticos, Compuestos Isociclicos Aromáticos, Editorial Atlante: México, 1956, vol. 2.

17. Furniss, B. S.; Hannaford, A. J.; Rogers, V.; Smith, P. W. G.; Tatchell, A. R.; Vogel's Textbook of Practical Organic Chemistry, 4ª ed., Longmann: London, 1979.

18. Gonçalves, D.; Wall, E.; Almeida, R. R.; Química Orgânica Experimental, McGraw-Hill: Rio de Janeiro, 1988.

19. Mano, E. B.; Seabra, A. P.; Práticas de Química Orgânica, Edart: São Paulo, 1976.

20. Wilkins, R.; Lowe, V. C.; J. Chem. Educ. 1979, 56, 488.

21. Moore, J. A.; Dalrymple D. A.; Rodig, O. R.; Experimental Methods in Organic Chemistry, $3^{\mathrm{a}}$ ed., Saunders College: Philadelphia, 1983.

22. Schotten, C.; Ber. Dtsch. Chem. Gess. 1884, 17, 2544; Baumann E.; Ber.Dtsch. Chem. Gess. 1886, 19, 3218.

23. Sugino, S.; Jpn. Patent 7485, 1954; Chem. Abstr. 1957, 50, 4236h.

24. Kozello, I. A.; Kinnelevskii, V. I.; Gasheva, A. Y.; Med. Prom. SSSR 1960, 14, 42. Chem. Abstr. 1960, 55, 3593c.

25. Tsukamoto, T.; Takabe, S.; Jpn. Patent 533,1959; Chem. Abstr. 1959, 54, 6770b. 
26. Behnish, R.; Henecka, H.; German Patent, 870,558, 1953; Chem. Abstr. 1953, 48, P2123b.

27. Katritzky, A. R.; Yousaf, S. I.; Can J. Chem. 1986, 64, 2087.

28. Träube, W.; Ber. Dtsch. Chem. Gess. 1893, 26, 2551; Träube, W.; Justus Liebig's Ann. Chem. 1923, 432, 266.

29. Kobayashi, E.; Yakagaku Zasshi 1962, 82, 445. Chem. Abstr. 1962, 58, 4552b.

30. Davis, T. L.; Clarke, H. T.; Phillips, R.; Org. Synth. 1941, Coll. I, 302; Davis, T. I.; Clarke, H. T.; Phillips, R.; Org. Synth. 1973, Coll. V, 589.

31. Sittig, M.; Pharmaceutical Manufacturing Encyclopedia, $2^{\text {nd }}$ ed., Noyes/ William Andrew Publishing, Park Ridge: New Jersey, 1988, vol. 2, p. 1441

32. Farmacopéia dos Estados Unidos do Brasil, $2^{\mathrm{a}}$ ed., Indústria Gráfica Siqueira: São Paulo, 1959, vol. 2, p. 24, 756.

33. U. S. Pharmacopoeia and \& National Formulary., United States Pharmacopoeia Publisher, Washington, D. C., 1995, USP23/NF18, versão em CD-ROM.

34. Bult, A.; Klasen, H. B.; Arch.Pharm. 1980, 313, 1016; Cai, E.; Kaoxue Tongbao 1981, 16, 27; Mastrolorenzo, A.; Scozzafava,A.; Supuran, C. T.; Eur. J. Pharm. Sci. 2000, 11, 99.
35. Federman Neto, A.; An. Assoc. Bras. Quim. 2000, 49, 107.

36. Perrin, D. D.; Armarego, W. L. F.; Purification of Laboratory Chemicals, $3^{\mathrm{a}}$ ed., Pergamon Press: New York, 1988, p. 94.

37. Saito, T.; Hayamizu, K.; Yanagisawa. M.; Yamamoto, O.; Wasada, N.; Someno, K.; Kinugasa, S.; Tanabe, K.; Tamura, T.; Tanabe, K.;.Hiraishi, J.; NIST - National Institute of Standards, AIST - Institute of Advanced Industrial Science and Technology, SDBS - Integrated Spectral Data Base System for Organic Compounds, disponível na Internet: www.aist.go.jp/ RIODB/SDBS/menu-e.html.

38. British Pharmacopoeia, $4^{\mathrm{a}}$ ed., The Stationary Office - TSO publisher, London, 1999, vol. 1, p. 844

39. Sinisterra, R. D.; Najjar, R.; Oliveira, L. F. C.; Spectrosc. Lett. 1993, 26, 305; Spectrosc. Lett. 1993, 26, 1813.

40. Topacli, C.; Topacli, A.; J. Mol. Struct. 2003, 654, 153

41. Federman Neto, A.; Freitas, S. C. S.; Daltin Jr., N.; Vichenewski, W.; Bonilha, J. B. S.; Borges, A. D. L.; Acta Scient. 2002, 24, 1591. 